\title{
Proceso enfermero aplicado a un paciente con úlceras por presión
}

\author{
Nursing process applied to a patient with pressure ulcers
}

\section{Processo de enfermagem aplicado a um paciente com úlcera por pressão}

\author{
Dora Luz Rodríguez Cruz \\ dorodriguez@uv.mx \\ ORCID: 0000-0001-8479-9742
}

Fabiola Cruz Núñez
facruz@uv.mx
ORCID: 0000-0002-0498-7645

\author{
Claudia Hernández Landaverde \\ claudihernandez@uv.mx \\ ORCID: 0000 - 0001-9010-9488
}

\author{
Blanca Judith Lavoignet Acosta \\ blavoignet@uv.mx \\ ORCID: 0000-0001-9501-5392
}

Universidad Veracruzana, México

Recibido 01 de octubre 2020 | Arbitrado y aceptado 14 de octubre 2020 | Publicado 22 de diciembre 2020

\begin{abstract}
RESUMEN
Las escaras (también llamadas úlceras por presión y úlceras de decúbito) son lesiones en la piel y el tejido inferior, resultan de una presión prolongada sobre la misma. En la mayoría de los casos, se manifiestan en la piel que recubre las partes óseas del cuerpo, como talones, tobillos, caderas y coxis. Las personas que corren mayor riesgo de tener escaras tienen limitada su capacidad para cambiar de posición o pasan la mayor parte del tiempo en una cama o una silla de ruedas. Pueden desarrollarse en el transcurso de horas o días, la mayoría se curan con tratamiento, pero algunas nunca se curan completamente. El proceso enfermero es una herramienta metodológica a través del cual Enfermería puede apoyar al paciente y/o familiar en brindar cuidados y educación en la prevención y atención de las úlceras por presión. En México, dos estudios metacéntricos reportan que la prevalencia cruda de UPP es del $12,94 \%$ y del $17 \%$, respectivamente. El presente caso se trata de una mujer adulto mayor, en edad extrema (81 años), atendida por su familiar, con regular adherencia a su tratamiento terapéutico y de cuidados, pasa la mayor parte del tiempo en cama, movilización regular aunque con buena higiene, con una escara de región sacra limpia en estadio II, la paciente y familiar muestra disponibilidad y motivación para aprender acciones de cuidado y prevención adoptar cambios en los hábitos de vida, ser más saludable además mejorar la salud familiar.
\end{abstract}

Palabras clave: Úlceras por presión; cuidados; enfermería; proceso enfermero; paciente

\begin{abstract}
Bedsores (also called pressure ulcers and pressure sores) are lesions on the skin and lower tissue, resulting from prolonged pressure on it. In most cases, they manifest on the skin that covers the bony parts of the body, such as heels, ankles, hips and coccyx. People who are most at risk for bedsores have limited ability to change position or spend most of their time in a bed or wheelchair. They can develop over the course of hours or days, most heal with treatment, but some are never completely cured. The nursing process is a methodological tool through which Nursing can support the patient and / or family in providing care and education in the prevention and care of pressure ulcers. In Mexico, two metacentric studies report that the crude prevalence of PU is $12.94 \%$ and $17 \%$, respectively. The present case is about an elderly woman, (81 years), cared for by her relative, with regular adherence to her therapeutic and care treatment, spends most of the time in bed, regular mobilization although with good hygiene, with a clean sacral region eschar in stage II, the patient and family member show availability and motivation to learn care and prevention actions, adopt changes in life habits, be healthier and improve family health.
\end{abstract}

Key words: Pressure ulcers; care; nursing; nursing process; patient
DR: Integrante del CA 459 Prácticas de Salud y Educación, Coordinadora del programa de Difusión de la Ciencia, la Cultura y el Deporte. Ejerce funciones de docencia, tutoría, gestión e investigación Producción científica en revistas indexadas, participa en ponencias nacionales. Jubilada del Instituto Mexicano del Seguro Social como sub Jefe de enfermería. Universidad Veracruzana. México.

CH: Integrante del CA 459 Prácticas de Salud y Educación, Ejerce funciones de docencia, tutoría, gestión e investigación, autora de artículos de investigación publicados a nivel nacional e internacional, ponente en congresos, certificada como ponente en congresos, certificada como
licenciada en Enfermería (con reconocimiento de idoneidad), Jefe de Servicio en el ISSSTE. Universidad Veracruzana. México.

FC: Líder del CA 459 Prácticas de Salud y Educación, Perfil ProDep, coordinadora del sistema Institucional de tutorías en la Facultad de Enfermería, integrante del NAB de la maestría en Enfermería, ejerce funciones de docencia, tutoría, gestión e investigación funge como directora $y$ jurado de tesis de pregra y posgrado. jurado de tesis de pregrado y posgrado. Universidad Veracruzana. México.

BL: Integrante del núcleo del CA 459 Prácticas de Salud y Educación, Perfil ProDep. Integrante de la comisión estatal de evaluación y rediseño curricular, miembro del NAB de la maestría en Enfermería coordinadora del Área Básica, integrante del Consejo Técnico responsable de certificación por la NOM ISO 90012015. Universidad Veracruzana. México. 
DR: Integrante del CA 459 Prácticas de Salud y Educación, Coordinadora del programa de Difusión de la Ciencia, la Cultura y el Deporte. Ejerce funciones de Cultura y el Deporte. Ejerce funciones de Producción científica en revistas indexadas, participa en ponencias nacionales. Jubilada del Instituto Mexicano del Seguro Social como sub Jefe de enfermería. Universidad Veracruzana. México.

CH: Integrante del CA 459 Prácticas de Salud y Educación, Ejerce funciones de docencia, tutoría, gestión e investigación, autora de artículos de investigación publicados a nivel nacional e internacional, ponente en congresos, certificada como licenciada en Enfermería (con reconocimiento de idoneidad), Jefe de Servicio en el ISSSTE. Universidad Veracruzana. México.

FC: Líder del CA 459 Prácticas de Salud y Educación, Perfil ProDep, coordinadora del sistema Institucional de tutorías en la Facultad de Enfermería, integrante del NAB de la maestría en Enfermería, ejerce funciones de docencia, tutoría, gestión e investigación, funge como directora y jurado de tesis de pregrado y posgrado. Universidad Veracruzana. México.

BL: Integrante del núcleo del CA 459 Prácticas de Salud y Educación, Perfil ProDep. Integrante de la comisión estatal de evaluación y rediseño curricular, miembro del NAB de la maestría en Enfermería, coordinadora del Área Básica, integrante del Consejo Técnico responsable de certificación por la NOM ISO 90012015. Universidad Veracruzana. México.

\section{RESUMO}

Escaras (também chamadas de úlceras de pressão e escaras) são lesões na pele e nos tecidos inferiores, resultantes de pressão prolongada sobre ela. Na maioria dos casos, eles se manifestam na pele que cobre as partes ósseas do corpo, como calcanhares, tornozelos, quadris e cóccix. Pessoas com maior risco de escaras têm capacidade limitada de mudar de posição ou de passar a maior parte do tempo em uma cama ou cadeira de rodas. Eles podem se desenvolver ao longo de horas ou dias, a maioria cura com tratamento, mas alguns nunca estão completamente curados. O processo de enfermagem é uma ferramenta metodológica por meio da qual a Enfermagem pode apoiar o paciente e / ou família na prestação de cuidados e educação na prevenção e cuidado das úlceras por pressão. No México, dois estudos metacêntricos relatam que a prevalência bruta de UP é de $12,94 \%$ e $17 \% 4$, respectivamente. O presente caso se refere a uma idosa, em extrema idade (81 anos), cuidada por seu familiar, com adesão regular ao seu tratamento terapêutico e assistencial, passa a maior parte do tempo acamada, mobilização regular, porém, com boa higiene com uma escara limpa da região sacral em estágio II, o paciente e seu familiar demonstram disponibilidade e motivação para aprender ações de cuidado e prevenção, adotar mudanças de hábitos de vida, ter mais saúde e melhorar a saúde da família.

Palavras-chave: Úlcera por pressão; cuidado; enfermagem; processo de enfermagem, paciente

\section{INTRODUCCIÓN}

$\mathrm{L}$ a enfermería en la actualidad y en nuestro país; al igual que en otras partes del mundo, lucha por consolidarse como una disciplina científica, convencidos de que los cuidados brindados por la mujer son milenarios y que distan de ser un oficio por su firme ideología (sistema de creencias y valores); y por estar cada día más inmersos en el mundo científico, requieren de una redefinición, que deje en claro lo que es la enfermería como profesión (1).

Ésta requiere de múltiples y variadas habilidades para adaptarse a las necesidades de las personas en los diversos contextos de la práctica profesional, lo que la hace ser creativa e innovadora, su razón de ser es atender la salud, a través de cuidados que permitan mantener y conservar la vida, mediante la satisfacción de las necesidades del individuo, familia y comunidad; por consiguiente, su campo de acción específico es la predicción, prevención y tratamiento de las respuestas humanas (1).

Además constituye el grupo de recurso humano más numeroso de los profesionales de la salud, en diversas competencias relacionadas con el cuidado de la salud. Florence Nightingale da inicio a la disciplina de enfermería, abordando con especial interés la observación como elemento primordial para brindar cuidado, desde allí, empieza su conceptualización como un pilar fundamental de Enfermería, es así como indirectamente se cimienta el Proceso de Enfermería (2).

Bajo ésta perspectiva, podría decirse que el Proceso de Enfermería, nace de la necesidad de las enfermeras de organizar la práctica del cuidado de una forma sistemática y científica, de manera que se logre satisfacer las necesidades de los 
usuarios en todos los ámbitos del ejercicio profesional de la disciplina, de forma oportuna, dinámica y medible (2).

Las(os) enfermeras(os) al aplicar el proceso, podrán experimentar satisfacción al ser valoradas(os) por los integrantes del equipo sanitario dadas sus diversas competencias profesionales; además de favorecer en ellas(os) el desarrollo del pensamiento crítico; es decir un pensamiento analítico, "(...) deliberado, cuidadoso y dirigido al logro de un objetivo", (Alfaro 1999:16) pues emplea "principios (...) y el método científico para emitir juicios basados en evidencias(...)" (Alfaro 1999:17). Por otra parte, el proceso compromete al individuo, familia y comunidad para tomar parte activa en las decisiones y cuidados que permitan mantener o recuperar la salud (1).

Es por esto que el proceso debe fundamentarse en una teoría científica, para éste trabajo se utilizó la teoría del déficit de autocuidado de Dorothea Orem, como un modelo general compuesto por tres teorías relacionadas entre sí, la teoría del autocuidado, la teoría del déficit de autocuidado y la teoría de los sistemas de enfermería, como un marco de referencia para la práctica, la educación y la gestión de la enfermería (3).

Ésta teoría establece los requisitos de autocuidado, además de ser un componente principal del modelo forma parte de la valoración del paciente. Donde los cuidados de enfermería son con el fin de ayudar al individuo a llevar a cabo y mantener acciones para conservar la salud y la vida, recuperarse de la enfermedad y afrontar las consecuencias de ésta, además, el entorno es entendido como todos aquellos factores físicos, químicos, biológicos y sociales, familiares o comunitarios, que pueden influir e interactuar en la persona; finalmente define la salud como un estado que, para la persona, significan cosas diferentes en sus distintos componentes y cambia a medida que cambian las características humanas y biológicas. La enfermera puede utilizar cinco métodos de ayuda, actuar compensando déficit, guiar, enseñar, apoyar y proporcionar un entorno para el desarrollo (4).

Por lo tanto al utilizar el proceso de atención de enfermería para llevar a cabo los cuidados de forma sistemática, la enfermera/o utiliza un pensamiento de análisis y crítica al planear el trabajo aplicado a los usuarios, su participación, de la familia y comunidad en las actividades influye en la evolución de mejoría y aprendizaje para un regreso a su entorno familiar, social y laboral con mejor calidad de vida además de la comunicación constante entre los profesionales de la enfermería para mantenerse informados y respaldar legalmente sus acciones.

Con todo esto la enfermería responde a un fenómeno mundial característico del presente siglo que es el envejecimiento poblacional. Según la Organización Mundial de la Salud, entre el 2015 y el 2030 la población con edad de 60 años o superior tendrá un crecimiento del $56 \%$, pasando de 901 millones a 1.4 billones. En México, de acuerdo con las cifras que estima el Consejo Nacional de Población, en 2017 habitaban en el país casi 13 millones de ancianos (5).

Aunado al avance científico y tecnológico y los cambios en los estilos de vida hacia un enfoque más saludable ha hecho que la esperanza de vida sea más larga conllevando en ellos nuevas enfermedades y/o complicaciones entre ellas las Úlceras por presión (UPP) en el adulto mayor, las cuales se identifican como: «úlceras de decúbito» y/o «escaras» son una lesión isquémica localizada en la piel y/o tejido subyacente con pérdida de sustancia cutánea; por lo general, se producen como resultado de la presión y/o fricción donde se localiza una prominencia ósea. Las UPP se 
producen debido a la presión aplicada al tejido blando, lo cual conlleva un flujo de sangre completa o parcialmente obstruido (6).

En la actualidad se ha incrementado notablemente el interés por el cuidado y abordaje de las Úlceras Por Presión, las cuales suponen un gran problema de salud, en el que la Enfermería juega un rol muy importante y con mayor independencia con respecto a otros campos. Éste interés, no solo se dirige hacia su tratamiento eficaz, sino que también hace especial hincapié en la prevención; ya que se estima que son evitables entre el 95-98\% de los casos. Además tienen gran repercusión en distintos ámbitos, están presentes en todos los niveles asistenciales, tanto a nivel primario, como en el secundario, afectando mayoritariamente a personas de edad avanzada, aunque no de manera exclusiva (7).

En México, dos estudios metacéntricos reportan que la prevalencia cruda de UPP es del $12,94 \%$ y del $17 \%$, respectivamente. En América Latina, Brasil presenta una prevalencia del $41,1 \%$, en tanto que en España es del 7,78. Como puede observarse, la presencia de UPP es un problema común en diferentes países. La prevención y el cuidado de UPP es responsabilidad del personal de enfermería, porque dentro de los cuidados básicos que proporciona a los pacientes está la movilización, la higiene, el cuidado de la piel y la prevención de lesiones, aspectos que están vinculados con la presencia de estas lesiones (8).

Con base en lo anterior, el gobierno mexicano, por medio del Programa Nacional de Salud 2007-2012, determinó cinco objetivos en la atención de la salud, los cuales estaban relacionados con la prestación de servicios de salud con calidad y seguridad. Uno de estos indicadores monitoreados desde 2006 fue el nivel de cumplimiento de acciones de enfermería para prevenir las UPP, de los resultados obtenidos han surgido propuestas de mejora, como la estandarización del uso de una escala de valoración, un protocolo para la prevención y el manejo, la implementación y la estandarización de las clínicas de heridas (9).

Ya que la principal causa de su formación es la presión ejercida y mantenida entre dos planos duros y la tolerancia de los tejidos a ésta. Por un lado tenemos el plano duro esquelético y prominencias óseas fisiológicas o deformantes del paciente y el otro plano duro generalmente externos a él, representado por la cama, silla, calzado u otros objetos (10).

La clasificación más utilizada se basa en la Conferencia de Consenso realizada en 1989 en Estados Unidos (Panel Nacional para Úlceras por Presión) y que en 1992 fue avalada por el Departamento de Salud y Servicios Humanos de ese mismo país.

- Grado I: eritema con piel indemne que no se blanquea con la presión. Aspecto clínico: eritema persistente, edema, despigmentación, calor local o induración de la piel sin ulceración.

- Grado II: pérdida cutánea de espesor parcial que involucra epidermis, dermis o ambas. Aspecto clínico: úlcera superficial tipo abrasión o vesícula.

- Grado III: pérdida cutánea de espesor completo que se extiende hasta la fascia sin comprometerla. Aspecto clínico: úlcera profunda con compromiso del tejido celular subcutáneo variable.

- Grado IV: pérdida cutánea de espesor completo que se extiende hasta el plano muscular, óseo o estructuras de soporte (por ejemplo: tendones, articulaciones). Aspecto clínico: úlcera profunda con necrosis extensa y destrucción de tejidos vecinos. En esta etapa pueden observarse trayectos fistulosos que amplían la 
extensión del área comprometida inicialmente (11).

La acción principal en las úlceras por presión es la prevención, reconocer a los pacientes de riesgo a través de varias escalas probadas que lo detectan, entre ellas encontramos la escala de Braden, Norton y Gosnell, siendo la de Braden la más utilizada. El manejo nutricional y el cuidado de la piel, haciendo una evaluación diaria de la misma por personal especializado en el manejo de heridas. También es importante educar y enseñar al paciente $\mathrm{y} / \mathrm{o}$ familiar para reintegrarlo a su entorno social y familiar educado y capacitado para llevar acciones en el cuidado de su salud.

\section{METODOLOGÍA}

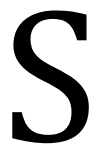
e realizó un estudio de caso clínico descriptivo, prospectivo $\mathrm{y}$ longitudinal, en el Hospital Regional Poza Rica, en el período comprendido de febrero-marzo 2020, a un adulto mayor con úlceras por presión en estadio ll, con daño tisular. Se estructuró de acuerdo con las etapas del proceso de atención de enfermería con el enfoque conceptual de la teoría de autocuidado de Dorothea Orem, aplicándose la guía de valoración que contiene los requisitos universales, requisitos de desarrollo y desviación de la salud, abordándose las áreas más afectadas en el paciente, se establecen diagnósticos y planes basados la interrelación taxonómica de la NANDA-NIC-NOC (12-14).

\section{DESCRIPCIÓN DEL CASO CLÍNICO}

M.J.R.M paciente femenina de 81 años de edad, adulta mayor cardiópata con DMII y HTA de 35 y 30 años de evolución respectivamente, insulinodependiente, hospitalizada en el servicio de MI el día 05 de
Septiembre con diagnóstico enfermedad cardiaca ateroesclerótica, ingresó a urgencias debido a que presento mareo y náusea al movilizarla del taxi a la silla de ruedas, perdió la consciencia durante un minuto con recuperación lenta, además de presentar relajación de esfínteres. Al momento de la exploración se encuentra orientada en tiempo, lugar y persona; no refiere disnea, ni palpitaciones o dolor torácico. La paciente percibe su estado de salud como "mal, esperando mejorar para poder vivir unos años más"; su edad de menarca fue a los 15 años y de menopausia a los 32 años. 4 embarazos previos, 2 partos y 2 abortos, con un intervalo de 3 años entre cada embarazo.

$\mathrm{Su}$ estilo de vida es sedentario, está postrada en cama la mayor parte del día y para comer o salir al patio ocupa su silla de ruedas; asiste a control médico periódico, se realiza detecciones oportunas y aplicación de vacunas de acuerdo a su esquema y edad. Lleva tratamiento médico para sus enfermedades crónicas degenerativas. Sin adicciones.

Vive con su hija mayor de 65 años, su nieta de 30 años y su bisnieta de 7. Profesa la religió católica y menciona no necesitar consejo de guía espiritual para la toma de decisiones, no utiliza tratamiento alternativo para sus padecimientos. Su principal apoyo en caso de problemas es su hija mayor, de quien depende económicamente. Ingiere comida balanceada, no practica ejercicio, duerme las horas necesarias para descansar; refiere que la comunicación dentro de su familia es buena, con quien más convive es con su bisnieta "la hace sentir activa". Su esposo falleció hace 50 años a causa de un coma diabético, su padre falleció de pulmonía hace 60 años.

La paciente refiere presentar dificultad para respirar cuando está en decúbito dorsal, mejorando con el cambio de posición 
aunque no necesita apoyo respiratorio. Cuenta con lesiones en la piel como hematomas en brazos a causa de una caída en su domicilio y UPP (II) en región sacra, sin datos aparentes de infección, de 3x3x.3cm de bordes regulares, limpia con 15 días de evolución. Hay dolor en miembros pélvicos, hormigueo, calambre y edema $(+)$.

Consume cerca de un litro de agua diario y considera que es acorde a sus necesidades, el agua proviene de garrafón. Su dieta en mayor frecuencia es a base de pollo, arroz, frijoles y carnes rojas. No usa prótesis dental faltan piezas dentales, con frecuencia presenta náuseas y reflujo.

Se le cambia 5 veces de pañal al día, según su hija la orina es color amarilla, con olor "fuerte por los medicamentos", por la noche un cambio de pañal. No requiere sonda vesical. Antes del ingreso hospitalario su patrón de evacuando era de 1 , de color café, de consistencia blanda semi-liquida y olor con flatulencia y presión rectal.

Para su movilización necesita apoyo, tanto para la cama, como la silla de ruedas. Tiene energía suficiente para comer, acostumbra descansar durante el día, en la noche duerme 8 horas aprox. Ha presentado cambios en su memoria, frecuentemente se le olvida lo que iba a realizar.

Ha sufrido cambios que han afectado parte de su vida ya que debe estar postrada en cama y no puede salir a pasear. Hay disminución de la agudeza auditiva que ha afectado su socialización "le desespera no saber de qué hablan", tiene aparato auditivo pero no lo usa porque le "incomoda", aunado a la dificultad para ver bien, le hicieron cirugía de cataratas en ambos ojos y colocaron lentes intraoculares hace unos años, pero actualmente presenta cataratas de nuevo.

Dentro de los episodios relevantes de su vida en su niñez su madre la abandonó, creció con su abuela materna, se casó muy joven (17 años). Durante su edad fértil la ausencia de su esposo y de adulto maduro su DM II.

Hace 7 años tuvo que cambiar su residencia, vivía junto con su hija en cerro azul, pero a raíz de que nació su bisnieta se fueron a vivir a su actual domicilio, dice "extrañar su casa y a sus vecinos".

- T/A 130/70 mmHg

- Peso: $90 \mathrm{Kg}$ Sat.02 96\%

- Temp axilar: $36.7^{\circ} \mathrm{C}$

- Talla: $160 \mathrm{cms}$.

- Glucosa capilar: 96 mg/dl FC: 72x'

- IMC: 35.16 FR: 18x'

\section{Exploración física:}

- Inspección general. Paciente femenina, con dolor en miembros pélvicos 7/10 EVA, estado nutricional IMC 35.16, complexión corporal y peso acorde a su estilo de vida, en posición semi fowler. Palidez de tegumentos, hematomas visibles en miembros superiores resultado de una caída, UPP en región sacra sin signos de infección. Afebril, consiente, orientada, asertiva.

- Cráneo sin alteraciones ni protuberancia, tamaño acorde y simétrico al cuerpo en correcta posición, pelo cano, fino.

- Dentadura incompleta con presencia de sarro y encías enrojecidas con halitosis.

- Cuello acorde a su biotipo, flexible sin dolor a los movimientos rotatorios, lateralización y extensión. Ganglios linfáticos no palpables, presencia de verrugas.

- Extremidades superiores integras, sin limitación a la movilidad, sin edema, hematomas en ambos brazos y ligeras laceraciones a causa de una caída previa.

- Extremidades inferiores integras, dolorosas, con presencia de edema (+) en ambas piernas, poca movilidad, venas varicosas visibles y palpables. Pies $\mathrm{y}$ dedos íntegros, uñas con presencia de Onicomicosis. 
Tabla 1. Razonamiento diagnóstico

\begin{tabular}{|c|c|c|c|}
\hline \multirow{2}{*}{$\begin{array}{c}\text { Requisito } \\
\text { Universal } \\
\text { Alterado } \\
\\
\text { A). Aire } \\
\text { Problema } \\
\text { identificado } \\
\text { P } \\
\end{array}$} & \multicolumn{3}{|c|}{$\begin{array}{c}\text { Dominio } 11 \\
\text { Seguridad y protección }\end{array}$} \\
\hline & $\begin{array}{c}\text { Etiología } \\
\text { E }\end{array}$ & $\begin{array}{c}\text { Sintomatología } \\
\mathrm{S}\end{array}$ & $\begin{array}{c}\text { Diagnóstico Enfermero } \\
\text { (NANDA) }\end{array}$ \\
\hline $\begin{array}{l}\text { Deterioro de la } \\
\text { integridad cutánea. }\end{array}$ & $\begin{array}{l}\text { Paciente senil (81 } \\
\text { años), poca } \\
\text { movilidad física, } \\
\text { uso de silla de } \\
\text { ruedas y cama } \\
\text { permanente, } \\
\text { diabetes mellitus } \\
\text { de } 35 \text { años de } \\
\text { evolución. }\end{array}$ & $\begin{array}{l}\text { Escara en región } \\
\text { sacra, bordes } \\
\text { regulares, limpia, } \\
\text { sin datos de } \\
\text { infección. }\end{array}$ & $\begin{array}{l}00046 \\
\text { Deterioro de la integridad } \\
\text { cutánea r/c edad extrema ( } 81 \\
\text { años), factores mecánicos, } \\
\text { alteración de la sensibilidad, } \\
\text { deterioro de la circulación, } \\
\text { presión sobre prominencias } \\
\text { óseas m/p alteración de la } \\
\text { integridad cutánea (Ulcera por } \\
\text { presión II) en región sacra de } \\
3 \times 3 \times 3 \mathrm{~cm} \text { de bordes regulares. }\end{array}$ \\
\hline
\end{tabular}

El requisito universal: afectado mantenimiento de un aporte suficiente de aire, sistema tegumentario en el dominio 11 de seguridad/protección se encontró de acuerdo a la taxonomía NANDA (2015-2017)

En el razonamiento diagnóstico (Tabla 1), se encontró como problema (P) deterioro de la integridad cutánea, causa (E) Paciente senil de 81 años, hematomas en miembros superiores a causa de caída reciente, poca movilidad física y uso de silla de ruedas y cama permanente. DMII de 35 años de evolución, Angina de pecho inestable con sintomatología (S) Ulcera por presión (II) en región sacra de $3 \times 3 \times 3 \mathrm{~cm}$ de bordes regulares, limpia sin datos aparentes de infección.

Por lo tanto, el requisito universal afectado mantenimiento de un aporte suficiente de aire, en el dominio 11 de seguridad/protección se encontró de acuerdo a la taxonomía NANDA (2015-2017) como diagnóstico enfermero "Deterioro de la integridad cutánea r/c los extremos de la vida (81 años), factores mecánicos, alteración de la sensibilidad, deterioro de la circulación, presión sobre prominencias óseas $\mathrm{m} / \mathrm{p}$ alteración de la integridad cutánea (Ulcera por presión (II) en región sacra de $3 \times 3 \times 3 \mathrm{~cm}$ de bordes regulares). 
Tabla 2. Plan de cuidados.

Diagnóstico enfermero: 00046 Deterioro de la integridad cutánea r/c edad extrema (81 años), factores mecánicos, alteración de la sensibilidad, deterioro de la circulación, presión sobre prominencias óseas $\mathrm{m} / \mathrm{p}$ alteración de la integridad cutánea (Ulcera por presión (II) en región sacra de $3 \times 3 \times 3 \mathrm{~cm}$ de bordes regulares).

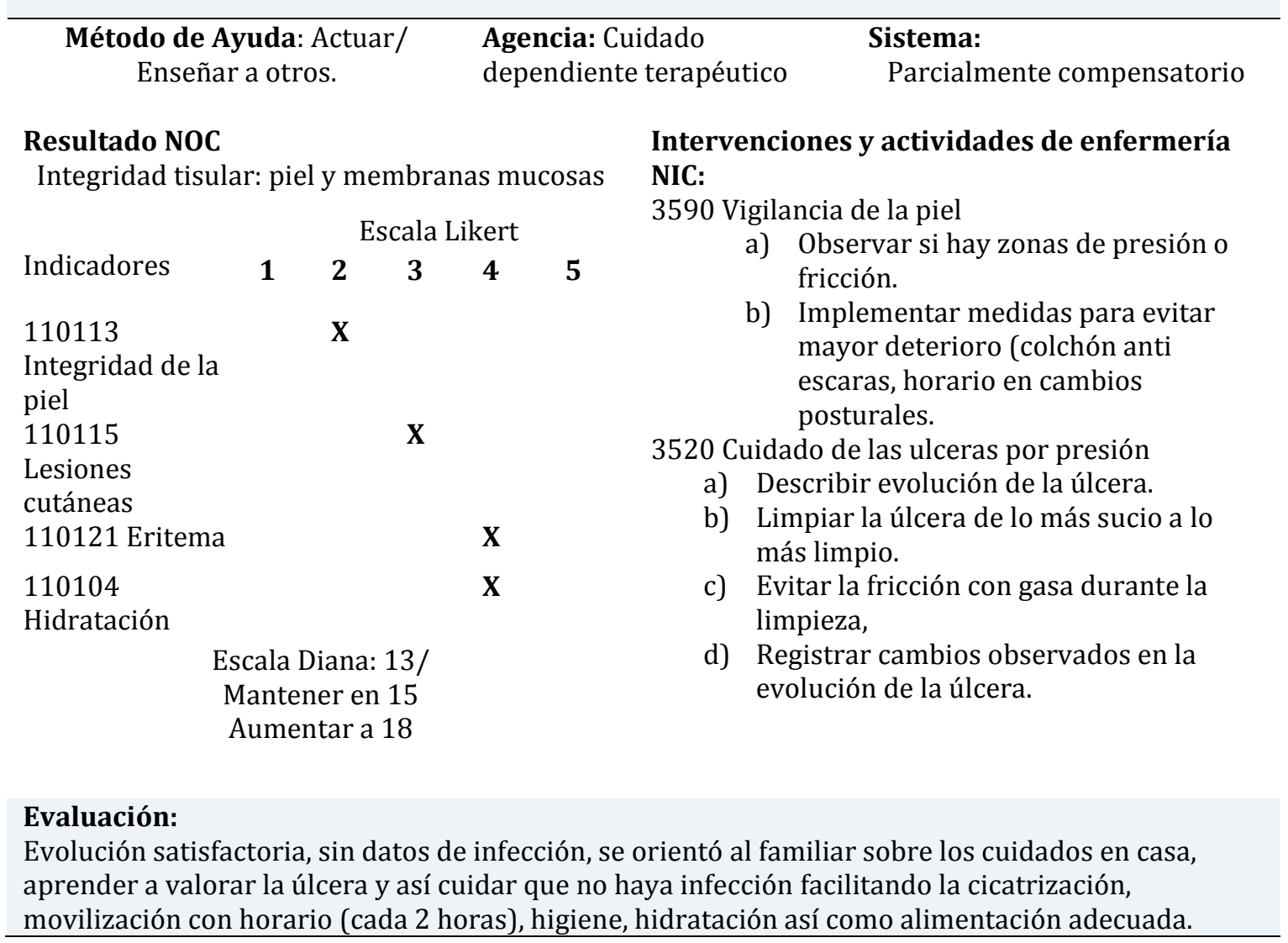

La planeación del diagnóstico de enfermería (Tabla 2): El método de ayuda fue el de actuar y enseñar a otros, la agencia que se utilizó cuidado dependiente terapéutico con un sistema parcialmente compensatorio. Los resultados (NOC) Integridad tisular: Piel y membranas mucosas. Con escala Likert se evaluaron los siguientes indicadores: Integridad de la piel con dos puntos, lesiones cutáneas tres puntos, eritema cuatro puntos, hidratación cuatro puntos, tomado en cuenta que a menor puntuación, mayor gravedad. Se encontró una escala diana de 13, para aumentar a 18 con las intervenciones (NIC) Vigilancia de la piel y sus actividades: Observar si hay zonas de presión o fricción, implementar medidas para evitar mayor deterioro (colchón anti escaras y cambios posturales con horario).La segunda intervención fue cuidados de la úlcera por presión, sus actividades: descripción de la evolución de la úlcera, curación de la úlcera de lo más sucio a lo más limpio, evitar la fricción con la gasa durante la limpieza y registrar los cambios observados en la evolución de la úlcera. En la evaluación que se realizó los resultados fueron óptimos ya que la herida evolucionó en forma satisfactoria, se orientó al familiar sobre los cuidados en casa, evaluar la úlcera y datos de infección, movilización con horario, higiene, hidratación y alimentación adecuada. 


\section{DISCUSIÓN}

$\mathrm{L}$ os pacientes que presentan úlceras por presión son aquellas que en la mayoría de los casos permanecen largo tiempo en cama, con enfermedades incapacitantes $\mathrm{o}$ adultos en edades extremas que no pueden llevar a cabo acciones de autocuidado como la movilización frecuente, los cambios de posición, estiramiento de la ropa de cama, más poco apoyo familiar, estos factores elevan la prevalencia, aunado esperanza de vida más larga y patologías recurrentes. Este tipo de lesiones causan malestar, dolor, incapacidad para adquirir determinadas posturas, el familiar $o$ cuidador debe llevar a cabo una higiene del paciente exhaustiva y diaria aunado a la curación de la herida, mantenerla siempre limpia. Además vigilar la dieta, consumir alimentos nutritivos, mantener al paciente en una cama limpia, libre de arrugas, sin humedad y con entorno agradable.

Enfermería juega un papel fundamental en estos pacientes otorgando cuidados, educación y capacitación al paciente y familiar, pero principalmente medidas preventivas para evitar su aparición y/ complicación de las mismas.

De acuerdo con un estudio latinoamericano de seguridad del paciente y la acreditación en salud (IBEAS) en el cual participó México se identificaron tres eventos adversos más frecuentes, entre ellos: neumonías nosocomiales, infecciones de heridas quirúrgicas y en tercer lugar las úlceras por presión he ahí la importancia de dar a conocer su prevalencia y la magnitud del problema para aplicar intervenciones de enfermería preventivas, de diagnóstico y tratamiento oportuno.

Fleck (2007) menciona que se deben incluir medidas generales para cualquier UPP (como cambios de posición frecuentes, cuidado de la piel y cuidados para corregir deficiencias nutricionales y sistémicas); asimismo recomienda la utilización de terapia húmeda con el objetivo de proporcionar un desbridamiento no traumático.

Referente a este caso clínico, la paciente y el familiar demostraron en todo momento disponibilidad e interés para el aprendizaje en acciones de cuidado, la paciente al presentar mejoría fue dada de alta a su domicilio donde se le dio seguimiento para evitar y prevenir complicaciones. Le herida mejoró con el involucramiento de su hija y cooperación de la paciente, actualmente está en vías de cicatrización en su domicilio, lo que nos demuestra que Enfermería puede renovar y actualizar la práctica diaria basándose en la ciencia, investigación y educación continua.

- Conflicto de intereses: Declaramos que el trabajo presentado para su publicación en esta Revista de Investigación en Salud VIVE, es original y no ha sido ni está actualmente en evaluación en ninguna revista ni congreso, igualmente nos hacemos responsables del contenido del mismo y manifestamos el acuerdo en que figuren nuestros nombres como autor y coautores. Por ultimo declaramos no tener ningún conflicto de interés en aquellas actividades que pudieran introducir sesgos en los resultados del trabajo.

- Financiación: El presente caso fue financiado por las investigadoras en su totalidad.

- Agradecimientos: Agradecemos a la Universidad Veracruzana, Facultad de Enfermería Región Poza Rica-Tuxpan por darnos las facilidades de la elaboración del presente trabajo. 
- También damos las gracias al Hospital Regional de SESVER por permitirnos llevar a cabo la investigación y aplicación del proceso al paciente en sus instalaciones.

\section{Investigación realizada considerando los tratados bioéticos}

En México la Ley General de Salud y el Reglamento de la Ley General de Salud en Materia de Investigación en Salud han basado parte de su articulado en la Declaración de Helsinki, lo cual es evidente en el contenido de la Ley General de Salud, Título Quinto, Investigación para la Salud, Artículo 100.

Por lo tanto el estudio se apegó a lo estipulado en la Ley General de Salud en el titulo quinto, Investigación para la Salud, Capitulo Único en el art. 100 que menciona:

I. Deberá adaptarse a los principios científicos y éticos que justifican la investigación médica, especialmente en lo que se refiere a su posible contribución a la solución de problemas de salud y al desarrollo de nuevos campos de la ciencia médica;

II. Podrá realizarse solo cuando el conocimiento que se pretenda producir no pueda obtenerse por otro medio idóneo.

III. Podrá efectuarse sólo cuando exista una razonable seguridad de que no expone a riesgos ni daños innecesarios al sujeto en experimentación;

IV. Se deberá contar con el consentimiento informado por escrito del sujeto en quien se realizará la investigación, o de su representante legal en caso de incapacidad legal de aquél, una vez enterado de los objetivos de la experimentación y de las posibles consecuencias positivas o negativas para su salud;
V. Sólo podrá realizarse por profesionales de la salud en instituciones médicas que actúen bajo la vigilancia de las autoridades sanitarias competentes.

En el Reglamento de la Ley General de Salud en Materia de Investigación para la Salud, Titulo segundo.-De los Aspectos Éticos de la Investigación en Seres Humanos, Capítulo I en los arts. 13. Disposición común.-en toda investigación en la que el ser humano sea sujeto de estudio, deberán prevalecer el criterio del respeto a su dignidad y la protección de sus derechos y bienestar;14, 17 (investigación sin riesgo-no se realiza ninguna intervención 0 modificación intencionada en las variables fisiológicas, psicológicas y sociales de los individuos que participan en el estudio) y 20 estipula que: se entiende por consentimiento informado el acuerdo por escrito, mediante el cual el sujeto de investigación o, en su caso, su representante legal autoriza su participación en la investigación, con pleno conocimiento de la naturaleza de los procedimientos y riesgos a los que se someterá, con la capacidad de libre elección y sin coacción alguna.

\section{REFERENCIAS BIBLIOGRÁFICAS}

1. Federación Mexicana de Colegios de Enfermería A.C.; Antología para el curso taller del proceso de enfermería. Compilación y actualización: comisión de certificación. 2013, Morelia, Pág. 159

2. Reina G. N. C. El proceso de enfermería: Instrumento para el cuidado. Umbral científico [internet]. 2010; (17):18-23, ISSN (Versión impresa): 1692-3375. Recuperado de: http://www.redalyc.org/articulo.oa?=3 0421294003

3. Naranjo HY, Concepción PJA, Rodríguez LM, Revisión Bibliográfica. La teoría Déficit de autocuidado: Dorothea 
Elizabeth Orem. Cuba: Gaceta Médica Espirituana Universidad de Ciencias Médicas. Sancti Spíritus [Internet].2017; (19), No. 3, ISSN 1608 - 8921, Recuperado de: http://revgmespirituana.sld.cu/index. php/gme/article/view/1129/pdf

4. Marcos EMP, Tizón B. E. Aplicación del modelo de Dorothea Orem ante un caso de una persona con dolor neoplásico. Gerokomos [Internet]. 2013; (24):168-177. ISSN 1134-928X, Recuperado de: http://scielo.isciii.es/scielo.php?pid=S 1134-

928X2013000400005\&script=sci_abst ract\&tlng=es

5. Ibarra TMC, Torres-Hernández E.A., Betancourt EMC, Prevalencia de los principales diagnósticos de enfermería en pacientes con demencia vascular según la taxonomía NANDA. Revisión bibliográfica. Rev Mex Enf. [Internet] 2019; (7):71-81. Recuperado de: http://www.innsz.mx/2018/RevistaEnf ermeria/RevistaEnfermeria-2019-2.pdf

6. Mijangos PMA, Puga C. C.L., Guillén L.C., Zúñiga CIR. El manejo de las úlceras por presión: intervenciones encaminadas a un oportuno manejo hospitalario. Evidencia Médica e Investigación en Salud. 2015; (8):2, 77-83 Recuperado de: https://www.medigraphic.com/pdfs/ evidencia/eo-2015/eo152e.pdf

7. Lucas AP, y Ferrer PMA. Diagnósticos de enfermería en úlceras por presión. [Tesis doctoral]. Facultad de Enfermería de Soria. Universidad de Valladolid. España: 2016. Recuperado en:

https://uvadoc.uva.es/bitstream/han dle/10324/20462/TFGO\%20857.pdf; sessionid=227B17B007162BAAB2CF5 C510B1E7F8A?sequence $=1$

8. Barrera AJE, Pedraza CMC, Pérez JG, Hernández JP, Reyes RJA, Padilla ZMP,
Prevalencia de úlceras por presión en un Hospital de tercer nivel en México. Gerokomos [Internet] 2016; 27 (4) 176-181 Versión impresa ISSN 1134928X, Recuperado de: http://scielo.isciii.es/scielo.php?script =sci_arttext $\&$ pid $=$ S1134928X2016000400009

9. Vela AG, Magnitud del evento adverso. Úlceras por presión. Coordinación Normativa de Enfermería de la Dirección General de Calidad y Educación en Salud (DGCES), Secretaría de Salud. México, Rev. Enfermería Instituto Mexicano del Seguro Social [Internet] 2013; 21 (1):3-8 Recuperado de: https://www.medigraphic.com/pdfs/ enfermeriaimss/eim2013/eim131b.pdf

10. Blanco LJL, Definición y clasificación de las úlceras por presión. Unidad de Lesionados Medulares Revisión de conjunto El Peu [internet] 2003; 23(4):194-198 Recuperado de: http://diposit.ub.edu/dspace/bitstrea m/2445/26068/1/545034.pdf

11. Prado A., Andrades P., Benítez $S$. Úlceras por Presión. En: editor Universidad de chile; Cirugía Plástica Esencial. Primera edición. Santiago de Chile: Editorial Hospital Clínico. 2005. p.111-126 ISBN 956-19-0477-2

12. Herdman TH, Katmisuru $S$. Diagnósticos Enfermeros definiciones y clasificación NANDA. 11 1 a ed. Barcelona. Elsevier: 2018-2020.

13. Butcher KH, Bulecheck MG, Dochterman MJ, Wagner. MCh. Clasificación de Intervenciones en Enfermería (NIC). 7a. ed. Barcelona. España: Elsevier; 2019.

14. Moorhead S, Swanson E, Johnson M, Maas ML. Clasificación de Resultados de Enfermería (NOC). 6ta. ed. Barcelona. España: Elsevier; 2019. 